REVISTA

de la

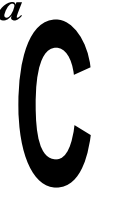

E

PAL

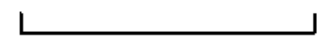

NUMERO 63

DICIEMBRE 1997

SANTIAGO DE CHILE

OSCAR ALTIMIR

Director

EUGENIO LAHERA

Secretario Técnico

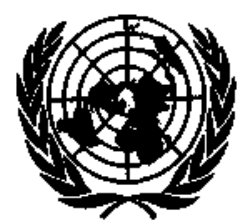

NACIONES UNIDAS 
El papel del sector público en el desarrollo latinoamericano

Ricardo Carciofi

La equidad en el presupuesto público

Juan Martin

Reformas a los sistemas de pensiones, mercado de capitales y ahorro

Andras Uthoff

Institucionalidad pública y políticas ambientales explícitas

e implícitas

Nicolo Gligo

La valoración de recursos naturales y ambientales no basada en el mercado en Centroamérica y el Caribe

Steve Shultz

Un modelo macroeconómico integrado para el Caribe

Lucio Vinhas de Souza

Virajes y derrapajes de la economía venezolana

José Miguel Benavente

¿Cuán no tradicionales son las exportaciones no tradicionales?

La experiencia de siete países de la Cuenca del Caribe

Alberto Gabriele

Apertura comercial y cambio estructural en la industria automotriz brasileña

Ruy de Quadros Carvalho, Sergio Robles Reis de Queiroz,

Flávia Luciane Consoni, loriara Costa y Janaína Pamplona da Costa

Historia evolutiva de una planta metalmecánica chilena

Jorge Katz y Héctor Vera

La importancia de la producción local y la pequeña empresa para el desarrollo de América Latina

Francisco Alburquerque

Publicaciones recientes de la CEPAL 


\section{Institucionalidad pública y políticas ambientales explícitas e implícitas}

\section{Nicolo Gligo}

Experto Principal

de Medio Ambiente,

CEPAL
En los últimos años el tema ambiental ha acrecentado su presencia en los análisis y propuestas sobre el desarrollo de los países de América Latina y el Caribe. Los graves procesos de desestabilización ecológica en el mundo, unidos a numerosas catástrofes y a la constante pérdida de recursos naturales, han contribuido a la toma de conciencia sobre esta dimensión. En mayor o menor medida, los gobiernos de todos los países de la región han procurado reforzar las políticas ambientales a través de diversas iniciativas legales, técnicas, institucionales y económicas. No obstante, aún falta mucho para superar los déficit existentes y para que las nuevas medidas que se pongan en práctica sean realmente eficientes y eficaces. Tras la inoperancia que se observa en relación con el tema del medio ambiente hay problemas mucho más complejos que la mayor o menor voluntad política o el grado de eficiencia burocrática de las estructuras ambientales. Este ensayo tiene por objeto examinar la compleja generación y aplicación de las políticas ambientales en los países de la región, analizando de partida la evolución de la estructura institucional pública, para colegir algunas recomendaciones que apunten a formas más novedosas y eficientes de encarar nuevas políticas ambientales y de conformar una institucionalidad ambiental pública que pueda ayudar a generarlas. 


\section{La evolución de las estructuras institucionales responsables de las políticas ambientales}

\section{Desde el término de la segunda guerra mun- dial hasta fines de los años setenta}

Los problemas ambientales siempre han estado presentes en la historia de la región (Gligo y Morello, 1982, pp. 112 a 148). Sin embargo, las categorías de análisis ambientales sólo comenzaron a aplicarse hace algunas décadas. Antes, el examen del medio ambiente se repartía entre los análisis de la calidad de vida de la gente y de la situación de los recursos naturales y se incluía tímidamente en algunas consideraciones sobre el deterioro de recursos derivado de los procesos de desarrollo.

La preocupación por los recursos naturales, especialmente los renovables, se remonta a la época de la Colonia. Varias crónicas de la época citan a gobernantes, naturalistas y clérigos que señalaron las desastrosas consecuencias de la conquista sobre los recursos naturales del territorio americano.

A comienzos del actual siglo, la explotación excesiva de algunos recursos naturales, la erosión de los suelos, la deforestación y la contaminación de aguas fueron señaladas reiteradamente por sus efectos negativos. Ya en ese entonces se afirmaba que los recursos naturales de la región no eran infinitos.

Sin embargo, fue a partir del término de la segunda guerra mundial cuando la concepción más sistémica del desarrollo empezó a tomar fuerza y el tema ambiental comenzó a preocupar a la gente y, por ende, a los estamentos políticos y los gobiernos. La preparación de la Conferencia Mundial sobre el Medio Humano, realizada en Estocolmo en 1972, y los posteriores acuerdos, sirvieron para que la temática ambiental fuese tratada y gestada globalmente.

Tras esa conferencia hubo una suerte de activismo ambiental en instituciones gubernamentales y se generaron políticas, leyes e instituciones dedicadas al medio ambiente. La pionera creación del Ministerio del Ambiente y de los Recursos Naturales Renovables (MARNR) en Venezuela marcó un hito en los países de la región y durante varios años, más que de modelo, sirvió de argumento para que los grupos y sectores de la institucionalidad pública de otros países lo mostraran como un ejemplo positivo.

No obstante los esfuerzos para darle al medio ambiente un tratamiento integral - como lo intentaba el enfoque holístico presente en un amplio estudio denominado "Sistemas ambientales venezolanos" (Venezuela, Ministerio del Ambiente y de los Recursos Renovables, 1982)_, en la mayoría de los países el tema ambiental se abordó a través de la planificación, o bien en forma sectorial, o bien en relación con la investigación de los recursos naturales.

Los enfoques basados en la planificación fueron aplicados por núcleos u oficinas que dependían de algún organismo de planificación, en una época en que ésta todavía seguía siendo importante en la región. Así lo hizo en Ecuador el Consejo Nacional de Desarrollo (CONADE), y en Paraguay la Secretaría Técnica de Planificación de la Presidencia de la República. En algunos países como Colombia y México, no obstante haberse ubicado las funciones ambientales en organismos sectoriales, los entes de planificación ayudaron a definir ciertas políticas ambientales, en particular referidas a regiones internas, sobre la base de programar la asignación de recursos.

La mayoría de los países centraron sus políticas ambientales en organismos sectoriales. La relación de las políticas espaciales con los organismos de desarrollo urbano hizo que algunos países ubicaran su organismo ambiental en el ministerio relacionado con el desarrollo urbano y la vivienda. En México, por ejemplo, el ente ambiental es la Subsecretaría de Ecología, dependiente de la Secretaría de Desarrollo Urbano y Ecología (SEDUE).

En otros países, como Argentina, la responsabilidad recayó en el ámbito de la salud, con la creación de la Subsecretaría de Ordenamiento Ambiental en la Secretaría de Salud y Medio Ambiente. El predominio del ordenamiento territorial y la ausencia del tema de la salud ligado al medio ambiente incidieron para que esta repartición se manejara con un alto grado de autonomía dentro del ministerio. 
Varios países optaron por reforzar o crear entidades en el sector silvoagropecuario. En Colombia el Instituto Nacional de Recursos Naturales y del Ambiente (INDERENA), depende del Ministerio de Agricultura pero tiene un alto grado de autonomía. Chile mantuvo importantes funciones ambientales en la Corporación Nacional Forestal, dependiente también del Ministerio de Agricultura. En Uruguay la responsabilidad ambiental correspondió al Ministerio de Agricultura y Pesca, principalmente a través de la Dirección de Suelos y Fertilizantes. En Guatemala, El Salvador y Honduras la solución también se centró en este ámbito. En Nicaragua, aunque el Instituto de Recursos Naturales y del Ambiente (IRENA) estaba definido como no sectorial, centraba sus acciones casi exclusivamente en la agricultura.

Los enfoques basados en la investigación de los recursos naturales se dieron en dos países: en Perú con la labor pionera de la Oficina Nacional de Evaluación de Recursos Naturales (ONERN) y en Chile a través del Instituto de Investigación de Recursos Naturales (IREN). Ambas instituciones impulsaron investigaciones integradas de recursos con planteamientos marcadamente ecosistémicos. En Bolivia, en esta etapa también se centró el examen del tema ambiental en la investigación, llevada a cabo por su Consejo Nacional de Investigación Científica y Tecnológica.

La complejidad de Brasil hace muy difícil definir sus improntas institucionales. En este país se dio una combinación de un ente centralizado, la Secretaría Especial del Medio Ambiente (SEMA), con organismos estaduales ubicados en diferentes esferas de la administración pública de cada estado. Algunos organismos federales ayudaron también a impulsar la incorporación explícita de la dimensión ambiental, entre otros, el Instituto Brasileiro de Desenvolvimento Forestal (IBDF), el Consejo Nacional de Desarrollo Científico y Tecnológico (CNPQ) y, en especial, el Instituto Nacional de Investigación de la Amazonia (INPA).

En la región latinoamericana fue muy importante el aporte de algunos proyectos con apoyo internacional para investigar el medio, planificar el desarrollo o estudiar algún proceso de deterioro. Estos proyectos, muchos de los cuales se realizaron también en los años ochenta, contribuyeron a arrojar luces sobre la situación ambiental. El Programa de Regionalización de Ecuador (ORSTOM-PROMARG); el programa ERTS de GEOBOL en Bolivia; el proyecto PNUD/FAO/BDF de investigación y desarrollo forestal en Brasil, que evaluó la deforestación de la Amazonia, y el proyecto ILPES/CDG de desarrollo de la cuenca del Río Guayas en Ecua- dor, son ejemplos claros de acciones que dinamizaron la incorporación del tema ambiental en algunos países de la región.

Además de estos proyectos, cabe destacar los estudios realizados por la Organización de los Estados Americanos (OEA) a través de su Departamento de Desarrollo Regional y Medio Ambiente, en especial los referidos a la cuenca del Plata; los proyectos de áreas protegidas de la Organización de las Naciones Unidas para la Alimentación y la Agricultura (FAO) y los estudios sobre desarrollo y medio ambiente realizados por la CEPAL, a través de su Unidad Conjunta CEPAL/PNUMA de Desarrollo y Medio Ambiente.

\section{La década perdida}

Si los años ochenta fueron la década perdida para la economía de América Latina y el Caribe, sin duda fue también una década muy negativa para la dimensión ambiental.

Los ajustes derivados de la crisis económica que golpeó a la región repercutieron notoriamente en las instituciones ambientales, que en ese entonces estaban empezando a emerger, eran débiles y carecían de recursos financieros a la altura de sus responsabilidades. La poca estatura política de estas nuevas estructuras llevó a que se las tratara de manera concordante con la ausencia de presión para favorecerlas.

Las restricciones presupuestarias y la consiguiente reducción de los gastos gubernamentales afectaron las partidas de gastos institucionales dirigidos a propósitos que, en la percepción de las autoridades nacionales, tenían baja prioridad económica (Brzovic, 1989). Y casi sin excepción las autoridades de los países de la región incluyeron el gasto ambiental entre los de baja prioridad.

Los efectos ambientales desencadenados por los instrumentos que buscaban reducir el gasto fueron múltiples y de diferente signo, lo que hace muy difícil determinar las relaciones de causalidad. Sin embargo, los efectos directos sobre las instituciones ambientales públicas fueron claramente identificables y negativos.

La restricción de los gastos corrientes de las reparticiones ambientales que controlaban la inversión sectorial limitó su capacidad de realizar o contratar estudios de impacto ambiental, o su capacidad de negociar y dar seguimiento a estudios de preinversión o vinculados a la ejecución de proyectos con consecuencias ambientales.

Todas las reducciones de gastos afectaron la capacidad de la institucionalidad ambiental vigente. Pero 
lo que es muy difícil de medir son sus efectos negativos en el ritmo de expansión de esta institucionalidad, que se planteaba como urgente por la creciente importancia del tema. No cabe duda de que los entes ambientales no gubernamentales, la opinión pública con conciencia ambiental crítica y los sectores ambientales de la administración pública, en cada uno de los países de la región, esperaban una notoria expansión en aspectos como reforzamiento institucional, nueva legislación, dotación de personal, recursos financieros, etc. Esta expansión no sólo no se obtuvo sino que, en la mayoría de los casos, hubo reducciones. Por ello, los efectos de la "década perdida" persisten hasta hoy, pues con las restricciones presupuestarias actuales, a los países les ha sido muy difícil superar este rezago.

A los problemas aludidos hay que sumar los efectos de una política de ajuste que en los países de la región, explícita o implícitamente, estableció que el medio ambiente no podía ser obstáculo para sus logros. Así, la institucionalidad ambiental vigente en ese entonces pasó a constituir una suerte de estorbo que debía ser en lo posible silenciado. En muchos países se diluyó la responsabilidad ambiental hasta el punto de no haber una autoridad clara; en otros, aunque ésta existía, era constantemente superada por acciones paralelas de los ministerios de economía o de los sectores productivos. Sin embargo, el peso de los procesos de deterioro, y también la preparación de informes nacionales para la Conferencia de las Naciones Unidas sobre el Medio Ambiente y el Desarrollo (Río de Janeiro, 1992), contribuyeron a fines de los ochenta y principios de los noventa a modificar las políticas vigentes y dar un nuevo impulso a las políticas ambientales y sus organismos.

\section{El impulso de los años noventa}

La conciencia ambiental cada vez mayor a nivel nacional y mundial impulsó una readecuación de los enfoques nacionales frente al tema. A la vez, la paulatina globalización de la economía creó nuevas formas de comercio internacional, en las cuales las consideraciones ambientales empezaron a tener un lugar insospechado.

La preparación de la Conferencia de Río de Janeiro tuvo variados efectos en los países de la región. En primer lugar, sirvió para que en distintos sectores y estamentos dentro de cada país hubiera una discusión profunda sobre los problemas del medio ambiente y los recursos naturales. Notable fue la movilización de científicos, académicos, personeros públicos, organismos ambientales no gubernamentales y otros que hizo Brasil para preparar su informe nacional. No en todos los países se dio esta discusión integral; algunos redujeron sus informes nacionales al solo aporte del organismo ambiental central.

En segundo lugar, la preparación de la conferencia sirvió para que se conceptualizaran las diferencias entre el norte y el sur. Los países de América Latina y el Caribe en su conjunto, y unidos a otras naciones en desarrollo, pudieron hacer un frente común para establecer que las soluciones a los problemas ambientales pasan indiscutiblemente por la erradicación de la pobreza (CEPAL, 1991; BID/PNUD, 1990).

Las nuevas relaciones comerciales entre los países del norte y los del sur ha sido uno de los factores que más ha pesado en la reacción de algunos países de la región frente a la temática ambiental. El caso de Chile resulta ilustrativo. Es indiscutible que la modalidad de desarrollo adoptada por este país no privilegia esta dimensión. Sin embargo, la amplia apertura de su economía ha creado frecuentes demandas ambientales, mucho más gravitantes que la posible presión interna desde los distintos estamentos de la sociedad chilena. Y no cabe duda de que la asociación con los países del MERCOSUR y el acuerdo de complementación comercial firmado con Canadá incrementarán estas demandas.

El Tratado de Libre Comercio de América del Norte y su Acuerdo Paralelo sobre Cooperación Ambiental, compromete a México a altos niveles de protección del medio ambiente. Una de las consecuencias más importantes de este tratado fue la creación de la Comisión de Cooperación Ambiental de América del Norte, cuyo consejo debe opinar sobre la compatibilidad de normas ambientales. La creación de la Comisión de Cooperación Ecológica Fronteriza (CCEF) también fue establecida por el Acuerdo Paralelo (México, Poder Ejecutivo Federal, 1996). El tratado ha ayudado a una mayor incorporación del tema ambiental y, en alguna medida, ha influido en la creación de un organismo central ambiental más fuerte; pero ha sido el único factor positivo para la dimensión ambiental en México. Los compromisos de este país con la Organización para la Cooperación y el Desarrollo Económicos en relación con el medio ambiente incluyen un número importante de decisiones que habrán de aplicarse.

En Centroamérica, los acuerdos de cooperación centroamericana incrementaron en los años noventa la preocupación por el medio ambiente. Se intensificaron las acciones de la Comisión Centroamericana de Ambiente y Desarrollo y se firmaron protocolos sobre desarrollo sustentable. 
La puesta en marcha del Mercosur ha privilegiado el aspecto comercial. Sin embargo, ha habido algunos avances ambientales que posiblemente se observen sobre todo en los efectos de las nuevas infraestructuras físicas de integración. La evaluación del impacto ambiental de la Hidrovía de la Cuenca del Plata se ha traducido en importantes avances en el conocimiento integrado de esa cuenca y los efectos de sus modificaciones.

El Pacto Amazónico ha seguido su tradición de privilegiar el conocimiento del ecosistema amazónico, su integración y los efectos de sus alteraciones.

Especial mención merece lo que sucede con la Agenda 21, emanada de la Conferencia de Río de Janeiro. Al evaluar lo que se ha hecho para aplicarla se observa que los países poco o nada la consideran. Si el país tiene que evaluar su seguimiento, lo que hace es recopilar información sobre el desarrollo que ha tenido el tema ambiental con posterioridad a la Conferencia de Río de Janeiro, considerando actividades que se hubieran realizado con o sin Agenda 21. La excepción son algunos acuerdos internacionales inscritos en la Agenda 21, que por su dinámica han desembocado en acciones derivadas de los compromisos adquiridos. Entre ellos se hallan los acuerdos sobre la capa de ozono y el cambio climático, amén de los avances en el Grupo Intergubernamental ad hoc sobre Bosques, de la Comisión sobre el Desarrollo Sostenible, de las Naciones Unidas.

\section{La nueva institucionalidad ambiental}

Los cambios producidos dentro de los países y la modificación del escenario mundial provocaron importantes transformaciones en la institucionalidad ambiental pública, la que se fortaleció en los países que crearon o reforzaron ministerios de medio ambiente, y también en los que radicaron la máxima autoridad ambiental en cuerpos colegiados, como comisiones o consejos. ${ }^{1}$ En algunos países se ha estructurado un "sistema institucional ambiental" que abarca las distintas instancias, niveles y desagregaciones; pero no pasa de ser una estructura configurada en el papel, ya que la temática sigue siendo abordada desde una institucionalidad central y, además, en el caso de países federativos, por instituciones estaduales o provinciales.

Lo tradicional en los países de la región es que la máxima categoría la otorga la condición de ministerio.

\footnotetext{
${ }^{1}$ Véase más detalles en PNUMA, 1996.
}

Por lo tanto, los esfuerzos se orientaron a crear ministerios que se hicieron cargo de los planteamientos sobre desarrollo sustentable. En general, este tema no se ha considerado una responsabilidad nacional compartida por todas las esferas; lo que ha predominado es la tendencia a que lo aborden organismos ambientales.

De los nuevos ministerios creados, algunos centran sus funciones no sólo en los problemas ambientales, sino que también hacen hincapié en la situación de los recursos naturales. Casi todos ellos tienen que ver en forma muy amplia con los problemas de la contaminación, pero en lo que hace a los recursos naturales, privilegian los renovables. Es el caso del ya tradicional Ministerio del Medio Ambiente y de los Recursos Naturales Renovables (MARNR) de Venezuela. Casos similares son el Ministerio del Medio Ambiente y Energía de Costa Rica; el Ministerio de Medio Ambiente y Recursos Naturales de El Salvador; la Secretaría de Estado en el Despacho de Recursos Naturales y Ambiente de Honduras, y el Ministerio del Ambiente y los Recursos Naturales (MARENA) de Nicaragua.

México creó hace dos años el Ministerio de Medio Ambiente, Recursos Naturales y Pesca, que no sólo tiene la responsabilidad ambiental tradicional, sino que abarca también sectores productivos o muy estrechamente ligados con éstos, a través de su Comisión Nacional de Aguas y su Subsecretaría de Pesca, y de programas productivos forestales de la Subsecretaría de Recursos Naturales.

Otros países que le han dado rango ministerial a la autoridad ambiental del país han llegado a similar solución, pero con algunas variantes. Brasil creó un Ministerio de Medio Ambiente y Amazonia Legal, que, además de abordar la problemática ambiental del país, privilegia el territorio amazónico que incluye los estados agrupados en la Amazonia Legal.

Para crear su Ministerio de Medio Ambiente, Colombia aprovechó las estructuras preexistentes de corporaciones autónomas de desarrollo regional, convirtiéndolas en corporaciones ambientales. El Ministerio recogió la experiencia del Instituto de los Recursos Naturales Renovables y del Ambiente (INDERENA), institución que ha entregado a América Latina y el Caribe aportes pioneros.

Algunos países de la región han encomendado el tema a un ministerio asociado a una actividad tradicional: en varios países del Caribe de habla inglesa son ministerios asociados a la salud y en otros al del turismo. En Centroamérica, Belice tiene un Ministerio de Turismo y Medio Ambiente. 
En Uruguay se ha seguido la antigua tradición de relacionar el tema ambiental con la planificación urbana y territorial; por ello se creó el Ministerio de Vivienda, Ordenamiento del Territorio y Medio Ambiente. En Cuba se le ligó a la ciencia y a la tecnología en el Ministerio de Ciencia, Tecnología y Medio Ambiente.

Dos países han tratado de hacer cambios institucionales para introducir el complejo tema del desarrollo sustentable. Bolivia fue pionero al crear el Ministerio de Desarrollo Sustentable y Medio Ambiente. Argentina transformó en 1997 la Secretaría de Recursos Naturales y Ambiente Humano en la Secretaría de Recursos Naturales y Desarrollo Sustentable.

Los países que optaron por comisiones o consejos privilegiaron las funciones de coordinación más que las de ejecución. Por ejemplo Ecuador, Guatemala y Chile tienen una Comisión Nacional de Medio Ambiente y Perú un Consejo Nacional de Medio Ambiente. Panamá ha centrado las funciones ambientales principalmente en el Instituto de Recursos Naturales Renovables (INRENARE).
El reforzamiento de la estructura institucional pública para el medio ambiente no se ha traducido en mayor eficiencia. En varios países de la región, las contradicciones ambientales propias de la modalidad de desarrollo predominante, que está en expansión, se han traducido en falta de voluntad política para impulsar el tema. A veces, el elevarlo al nivel de ministerio o de comisión nacional ha sido una operación cosmética. Para varios gobiernos el medio ambiente es un obstáculo, un personaje no invitado a la fiesta del crecimiento económico con el que hay que compartir recursos a regañadientes, al que hay que aislar o arrinconar usando formas elegantes. Y una de ellas puede ser un ministerio que debe ajustarse a leyes ineficaces e ineficientes o que tiene muy pocos recursos.

No obstante, existen gobiernos en los cuales la dimensión ambiental empieza a ocupar un lugar de importancia, y las decisiones de sus organismos ambientales comienzan a tener la fuerza necesaria como para modificar seriamente la política ambiental de sus países.

\section{II}

\section{Políticas ambientales explícitas y políticas ambientales implícitas} en las políticas económicas

\section{Las contradicciones del reforzamiento institucional}

Si se analizan las variadas medidas que se han tomado en los últimos años en relación con el medio ambiente y los recursos naturales, especialmente ligadas al reforzamiento institucional del tema ambiental en el aparato público, habría que concluir que se ha avanzado en la incorporación de la dimensión ambiental en el quehacer de los países. A los notorios cambios de la estructura pública antes descritos hay que sumar, entre otras medidas, nuevas legislaciones y reglamentos, mayores controles y normas, planes de restauración ambiental, ampliación de las áreas protegidas y reforzamiento de su gestión, programas para mitigar el impacto de grandes proyectos, programas de capacitación y de educación ambiental y compromisos ambientales internacionales.
Sin embargo, y las cifras así lo confirman, no existe hoy ningún indicador o estadística que señale que la situación ambiental en la región ha mejorado. Por el contrario, todas las estadísticas muestran que el deterioro ha continuado (Gligo, 1995, pp. 107 a 122; PNUMA, 1997, pp.80 a 95).

Todo parece indicar que los esfuerzos desplegados en la región para revertir las tendencias negativas, que han sido muchos, sólo han servido para que determinados procesos deteriorantes atenúen sus pendientes negativas, sin modificar sus signos.

Esta situación suele atribuirse a la ineficiencia del aparato burocrático público, a la falta de compromisos de los sectores empresariales, o, en forma más genérica, a la insuficiente conciencia ambiental de la población.

Sin embargo, las causas son mucho más complejas: la interacción de diversos factores como las con- 
tradicciones ambientales del modelo económico —en particular de los horizontes de planificación-y los conflictos entre los bienes sociales y privados; las distintas racionalidades de los actores sociales, especialmente de los productivos; la coexistencia de distintos grados y tipos de conciencia ambiental en la población (desde difusa a crítica), y la tardía maduración política de los estamentos tradicionales de los poderes legistativos.

Existe un aspecto clave que debería, de alguna manera, integrar y jerarquizar factores que redunden en una gestión capaz de provocar un cambio de signo en los procesos ambientales deteriorantes: la forma en que se generan las políticas ambientales públicas derivadas de la aplicación de la legislación vigente y de los programas de gobierno. Hasta ahora no se ha reconocido debidamente la importancia de este factor para impulsar la incorporación del tema ambiental.

La política ambiental explícita se origina en los organismos centrales ambientales de la administración pública: los ministerios de medio ambiente o las comisiones o consejos ambientales. Pero hay políticas ambientales implícitas que surgen de los ministerios o del poder central, relacionadas casi todas con el crecimiento económico y cuya trascendencia no ha sido debidamente sopesada. Las políticas de crecimiento económico y las políticas sociales son las que mandan en los países y como suelen privilegiar el corto sobre el largo plazo, generan políticas ambientales implícitas de signo negativo.

No hay que confundir las políticas ambientales implícitas en las políticas de desarrollo con los impactos ambientales de ellas. Cuando se habla de políticas ambientales implícitas se plantea que éstas son medianamente conocidas y que requieren una decisión previa de carácter político o técnico derivada de las políticas de desarrollo.

\section{Las políticas ambientales explícitas}

Las políticas ambientales explícitas son por lo general reactivas. Tratan de disminuir los efectos negativos que se generan en los procesos de producción y en el consumo, debido a los efectos de la modalidad de desarrollo prevaleciente.

Casi todos los organismos públicos ambientales de los países de la región son reactivos en sus políticas ambientales. Responden a las urgencias que se derivan principalmente de la contaminación producida por la expansión urbana e industrial, la deforestación, la erosión de los suelos, el deterioro de los re- cursos del mar y la contaminación por la actividad minera.

Existen fuerzas subyacentes que van mucho más allá de la voluntad política de los gobernantes, la ideología de éstos o la conciencia ambiental de la población, por causas que se arrastran desde hace siglos.

Uno de los factores subyacentes de más peso es el incremento de la población. Aunque la región está en una etapa definida como de "transición demográfica", el incremento poblacional incide en el aumento de las demandas económicas y sociales, con el consiguiente impacto ambiental.

El otro factor subyacente es la persistencia histórica de la inequidad y de la pobreza. La persistencia de la pobreza crea una mentalidad de sobrevivencia y ésta a su vez condiciona una racionalidad de muy corto plazo que no lleva a invertir para las futuras generaciones.

Las iniciativas más novedosas de las políticas ambientales explícitas son las que tienen que ver con los sistemas de evaluación del impacto ambiental que casi todos los países han puesto en funcionamiento. Aunque la evaluación del impacto ambiental podría aparecer como no reactiva, su aplicación claramente lo es. Normalmente no se evalúan políticas y programas, sino proyectos decididos previamente en algún sector de la economía que no se define como ambiental. Estas evaluaciones sólo buscan aminorar los impactos; son pocas las ocasiones en que logran modificar sustancialmente un proyecto y menos aún las de rechazarlo.

Otras políticas ambientales explícitas dicen relación con la conservación de los recursos naturales renovables. Estas son claramente reactivas. Un ejemplo clásico son las políticas destinadas a reducir la deforestación, que en la región no han tenido éxito. Casi todos los países cuentan con legislación e instituciones para la protección forestal, las que deberían definir las políticas ambientales explícitas. No obstante, los factores que inciden en la deforestación escapan al control de estas políticas explícitas. Así, los procesos de expansión de la frontera agropecuaria generan el uso de sistemas de tumba, roza y quema, que tienen un altísimo costo ecológico. Por otra parte, las políticas de precios de la energía repercuten en una menor o mayor presión para producir leña proveniente de los bosques nativos.

También han fracasado las políticas de conservación del suelo, ya que la erosión, el agotamiento de nutrientes y la salinización están presentes en todos los países de la región. 
Las políticas de conservación de los recursos del mar derivan de las amenazas de crisis sociales por disminución de la actividad pesquera y de las advertencias de los científicos que estudian los recursos marinos. Como es difícil que la opinión pública tome conciencia de los problemas de disminución de estos recursos, la reactividad es menor que en los casos anteriores.

Las políticas ambientales explícitas referidas a la calidad de vida de la población, especialmente las relacionadas con la salud (como la contaminación), emanan de los problemas cotidianos que enfrentan las personas en cuanto al consumo y en el trabajo. Posiblemente son las más reactivas, por la presión que ejerce la población; desafortunadamente, aún falta mucho para que ésta exija políticas preventivas más estructuradas.

En términos generales, se aprecia que las políticas ambientales explícitas han tenido poco éxito. Pese al reforzamiento institucional de los organismos ambientales del sector público, concebidos como organismos ambientales reactivos, éstos han navegado casi sin excepción en aguas muy difíciles y en su mayoría han enfrentado serios conflictos con otras instancias de la burocracia estatal y también con la sociedad civil, especialmente los grupos empresariales y los organismos no gubernamentales ambientalistas.

Las funciones otorgadas a estas estructuras gubernamentales han sido claramente limitadas y no han podido permear en los sectores de la economía ligados a la actividad productiva.

\section{Las políticas ambientales implícitas en las políticas públicas}

El poco éxito de las políticas ambientales generadas en los organismos ambientales centrales se ha debido entonces a su condición de reactivas frente a modalidades de desarrollo con altos costos ambientales.

Los organismos ambientales de los países de la región muestran preocupación cuando el producto interno bruto crece, ya que una tasa alta se traduce en más automóviles, más actividad industrial y más residuos. Pero no sólo les preocupa el incremento del consumo sino también las modalidades de producción. Así, una tasa de inversión alta se puede convertir en una amenaza para los recursos del mar, para los bosques nativos o para los recursos hídricos.

Es obvio que las decisiones que se toman en otros ámbitos de la política pública o en los sectores productivos significan medidas que influyen en la transfor- mación del medio ambiente y constituyen políticas ambientales implícitas. Desafortunadamente, hasta la fecha se ha reflexionado poco sobre este tipo de políticas.

La crisis económica de los años ochenta y las medidas de ajuste que ella generó crearon una suerte de política ambiental implícita que, en términos globales, trata de minimizar la problemática ambiental. Si se analiza la aplicación de los tratamientos de shock utilizados en diversos países de la región, se comprueba que cuanto más severos han sido menos importancia se le ha dado al medio ambiente. Para los gobiernos que han aplicado las recetas anticrisis, el tema ambiental es molesto y sólo se le incorpora al debate cuando es un factor de negociación en un acuerdo comercial bilateral o multilateral, o cuando interfiere con la comercialización de algún producto.

Más atrás se hizo referencia a los efectos de las políticas de reasignación del gasto y de reducción de la demanda agregada en las instituciones ambientales públicas. Es importante analizar cuáles fueron las políticas ambientales implícitas en esas políticas económicas.

\section{a) Políticas macroeconómicas}

Las políticas macroeconómicas repercuten en la situación ambiental al mantener o extender una modalidad de desarrollo abiertamente perjudicial para el medio ambiente. No existen análisis a fondo de las políticas ambientales que derivan de ellas. Tres son las políticas que, desde una perspectiva global, los países tienen interés en profundizar.

i) Política de fomento de las exportaciones. Hasta la fecha no se aprecia en este ámbito una preocupación por conservar los recursos naturales. Al contrario, dada la creciente globalización de las economías, los países de la región tratan de fomentar sus exportaciones a toda costa, con medidas de estímulo y apoyo a través de instituciones encargadas del fomento exportador. Si existen consideraciones ambientales ellas se deben a las demandas de los países importadores.

ii) Política de captación de inversiones extranjeras. Para captar esos capitales casi todos los países tratan de dar una imagen de estabilidad económica y destacar lo baratos que son sus recursos naturales. El concepto de baratura se debe, por lo general, a que no se incorporan los costos ambientales y, a veces, al ofrecimiento de desgravámenes. Hay países que al dar a conocer las facilidades que se otorga a la inversión extranjera destacan entre ellas los escasos controles 
ambientales. En ciertos casos la publicidad ha ido más allá de lo que se podía ofrecer conforme a restricciones legales vigentes, lo que ha generado conflictos serios una vez que la inversión se ha hecho.

iii) Política de fomento de la ocupación del espacio. Muchos de los territorios de América Latina se consideran aún no ocupados y aptos para tener actividades económicas. En varios países, por necesidades económicas o por razones geopolíticas, se ha impulsado deliberadamente la ocupación masiva de sus territorios. Los estímulos han sido económicos: desgravámenes para actividades productivas como la ganadería; creación de áreas de radicación industrial con ventajas fiscales; construcción de infraestructura de transporte para posibilitar la incorporación territorial, y otros. Todas las políticas ambientales implícitas que han derivado de estos estímulos han tenido un fuerte impacto negativo.

\section{b) Las políticas económicas sectoriales}

Estas políticas generan políticas ambientales implícitas de la mayor importancia. Los sectores productivos de la economía condicionan la inversión, el crecimiento económico y la transformación del medio ambiente. Son ellos sectores que tienen responsabilidades por los residuos que se generan en las actividades productivas que fomentan, o en el consumo de los productos.

i) Las politicas de desarrollo industrial, que en la región tienen diversos grados de eficacia, generan un conjunto de políticas ambientales implícitas derivadas de varios factores (insumos utilizados, procesos de transformación, generación de residuos, destino de los productos, etc), las que en muchos casos tienen signo negativo. En temas como la bíodegradabilidad de los productos, sus vidas útiles y el reciclaje existe sin duda un notorio déficit en la política ambiental referida a la industria.

ii) Las políticas de desarrollo energético han incorporado en alguna medida el tema ambiental. La temprana maduración de los problemas ambientales derivados de las fuentes energéticas, como los efectos globales del consumo energético en la atmósfera, han llevado en la región a incorporar en cierto grado la dimensión ambiental en la planificación y gestión de la producción de energía. Desde hace varias décadas se estudian los impactos ambientales de los grandes emprendimientos hidroeléctricos, configurando políticas ambientales explícitas (por ejemplo, en los casos de Salto Grande, Itaipú y Yaciretá-Apipé, donde se logró implementar ciertas medidas mitigantes). Des- afortunadamente, las decisiones de incluir el aspecto ambiental suelen tomarse después de definir el lugar de construcción, la capacidad instalada y el diseño macro, lo que genera altos costos ecológicos que podrían haberse evitado. Por otra parte, a las preocupaciones tradicionales por el impacto de fuentes energéticas como la biomasa, las represas hídricas y la energía nuclear, cabe añadir las relacionadas con la eficiencia energética y los patrones de consumo, que deberían rescatarse.

iii) Las políticas agrícolas tienen gran importancia ambiental porque la agricultura transforma ecosistemas vivos. La expansión de la frontera agropecuaria (proceso apoyado por varios países mediante medidas como desgravámenes, apertura de carreteras y créditos agrícolas), así como el fomento del desmonte que hacen algunos países con subsidios para el uso de maquinaria o con créditos para los cuales se exige un porcentaje del predio deforestado y limpio, tienen implícito un alto costo ecológico en deforestación, pérdida de biodiversidad, empobrecimiento de suelos, erosión y embancamiento de cursos y espejos de agua. Por su lado, la modernización agrícola, entendida como la aplicación de tecnologías de artificialización generalmente alta del ecosistema, conlleva políticas ambientales implícitas. Las políticas de modernización tienden a ser de corto plazo, ya que crean agrosistemas de baja estabilidad natural. El objetivo de lograr una alta productividad en el corto plazo plantea contradicciones ambientales de muy difícil solución. Las políticas ambientales implícitas en un porcentaje importante de planes y programas de desarrollo agrícola son de signo negativo, ya que en ellas se externalizan los costos ecológicos de los principales procesos de deterioro que afectan al agro, como la erosión y el agotamiento y salinización de los suelos.

iv) Las políticas de reforestación y explotación forestal, dadas a través de créditos, subsidios u otras vías, no obstante la exigencia de que, a través de las políticas ambientales explícitas de regulación y control se les exige que sean sustentables, generan políticas ambientales implícitas negativas. El caso más ilustrativo son las políticas de reforestación que provocan una sustitución del bosque nativo por plantaciones. El bosque nativo tiene funciones: de regulador hídrico, habitat de la biodiversidad, freno a la erosión del suelo, aporte al paisaje, etc, las que se pierden irremediablemente, ya que las plantaciones sólo aportan una mínima parte de ellas.

v) Las políticas de obras públicas conllevan políticas ambientales implícitas importantes. Los cami- 
nos, puentes, embalses, canales de riego, muelles y puertos, se traducen en transformaciones significativas del medio ambiente $\mathrm{y}$, aunque muchas de ellas se realizan con estudios de impacto ambiental, éstos son sólo correctivos. Cuando la ingeniería transforma el medio ambiente persigue que las modificaciones sean beneficiosas y sustentables en el tiempo. Generalmente esto se logra, pero en algunos casos la artificialización es negativa. En efecto, con frecuencia se producen pérdidas de infraestructura, en particular caminos y obras anexas, debidas a políticas de expansión que contienen implícitas políticas ambientales de signo negativo, porque dan baja ponderación a factores climáticos, movimientos telúricos, etc.

vi) Especial mención se debe hacer de las politicas de desarrollo científico y tecnológico. Por una parte, la investigación científica de nuestro territorio tiene serios rezagos, sobre todo en materia de biodiversidad. Por otra parte, la investigación tecnológica relacionada con el medio ambiente tiende a hacerse sólo respecto de tecnologías limpias, sin explorar a fondo los impactos ambientales de las tecnologías de uso corriente. Además, se ha descuidado el análisis de nuevos tipos de tecnologías que permiten cosechas cada vez mayores de recursos naturales.

vii) Las políticas de desarrollo urbano muestran la complejidad de un tema con serios rezagos en la región. En ella se entremezclan políticas de ordenamiento urbanístico, de vivienda, de transporte, de industria, de centros de recreación, servicios y consumo, y de parques y jardines. Todas envuelven planteamientos ambientales y su aplicación tiene notables repercusiones en el medio ambiente. Las políticas regulatorias que adoptan las ciudades generan políticas ambientales implícitas que se articulan en distintos grados con las políticas ambientales urbanas explícitas. En algunos casos la articulación es adecuada, pero en otros existen francas contradicciones (por ejemplo, entre la política de enajenación de parques para ampliación vial o construcción de viviendas y la política de creación y mejoramiento de parques y jardines). Como la evolución de las grandes ciudades está estrechamente ligada a los procesos de desarrollo industrial la política ambiental implícita derivada de la localización industrial debería ser debidamente jerarquizada en función del ordenamiento ambiental del territorio. Desafortunadamente, en la región las políticas de localización ambiental suelen ser un subproducto de los planes reguladores de las ciudades y responder poco a consideraciones ambientales, salvo en lo que se refiere a la disponibilidad de ciertos recursos naturales — como el agua - y en los planes de localización ambiental suelen privilegiarse los costos de transporte, la disponibilidad de servicios, el acceso a mano de obra calificada, etc. Por lo demás, la ciudad articula bienes antrópicos en un medio natural dado. La complejidad de las estructuras y de los flujos de materiales, energía e información con frecuencia lleva a minimizar la importancia del medio natural, y a generar políticas urbanas implícitas de alto costo ambiental. Así, muchas de las políticas de expansión urbana en la región no obedecen a criterios ambientales, sino a presiones por la disponibilidad de terrenos baratos y por la especulación con el suelo urbano; asimismo, la construcción de vías para automóviles en desmedro de las vías preferenciales para la locomoción colectiva genera congestión y contaminación.

\section{III}

\section{Hacia políticas ambientales eficaces}

El hecho de que se rescate la importancia de las políticas ambientales implícitas en las políticas económicas, y en general en las políticas de desarrollo, no quiere decir que ellas sean alternativas a las políticas ambientales explícitas generadas por los organismos ambientales. Al contrarío, ambos tipos de políticas se complementan.

De partida hay que suponer que las políticas generadas en los organismos ambientales son reactivas, debido al alto costo ambiental que tiene la aplicación de las modalidades de desarrollo predominantes en la región. Para lograr la solución ideal se requeriría transformar esas modalidades de desarrollo para hacerlas sustentables, pero como esta utopía está aún bastante lejos, hay que tratar en forma pragmática de avanzar lo más posible con soluciones intermedias para modificar la tendencia actual.

Lo ideal sería lograr que las políticas ambientales implícitas en las políticas públicas fuesen de signo positivo y contribuyesen así a una gestión del desarrollo ambientalmente sustentable. Este logro, hoy utópico, debería ser la condición esencial para estructurar 
el sistema ambiental de un país sobre la base de comisiones o consejos solamente coordinadores. Su ausencia explica los fracasos y tropiezos de varias comisiones nacionales. Por esta razón, se recomienda buscar soluciones realistas que crucen las políticas explícitas e implícitas, buscando su complementariedad y una expresión institucional referida al sector público que sea coherente con este cruzamiento.

Veremos a continuación cuáles serían entonces los aspectos que es más importante reforzar en la matriz derivada del cruce entre políticas implícitas y explícitas.

\section{El cruce de las políticas ambientales implícitas con las explícitas}

En la matriz mencionada, el casillero que aparece casi vacío en la mayoría de los países de la región es el que cruza el ordenamiento ambiental territorial (política explícita) con el desarrollo regional (política implícita). La expresión espacial de la imagen-objetivo del territorio de cada país, y su consecuente cuantificación, permite proyectar desagregadamente las transformaciones sociales y ambientales y facilita el tratamiento de conflictos sociales y ambientales. Además, a fin de configurar esta imagen-objetivo, el ordenamiento ambiental territorial no sólo se cruza con el desarrollo regional, sino que para lograr más eficacia debe cruzarse también con todos los sectores productivos.

Otro cruce que es necesario efectuar es el de la política de fomento de las exportaciones (política implícita) con la de evaluación del impacto ambiental (política explícita), dada la presión sobre ciertos recursos, en especial los renovables, en una economía que privilegia las exportaciones.

Los procesos que se dan en el espacio rural son básicos para la conservación de los recursos naturales renovables. Por esta razón, las políticas ambientales implícitas en las políticas de desarrollo rural debieran tener mayor jerarquía. Entre ellas tiene especial relevancia la expansión de la frontera agropecuaria, la que se debe cruzar con varias política explícitas, a saber: las políticas de lucha contra la deforestación, la erosión y la desertificación, además de las de conservación de la biodiversidad.

En cuanto a los sectores económicos, se reitera la importancia de sus políticas implícitas y la necesidad de incorporar más la dimensión ambiental en ellas. Es preciso que los distintos sectores de la economía se incorporen a los intentos de ordenamiento ambiental territorial y que junto con intensificar las evaluaciones de impacto ambiental (en la mayoría de los casos se analizan sólo los grandes proyectos), se consideren los impactos de los programas y las líneas de acción.

La búsqueda de soluciones para abordar el problema ambiental, más allá de estas consideraciones generales, debe ser una empresa de cada país. Aunque hay características comunes a todos los países, ellos presentan diferencias derivadas no sólo del grado de maduración del tema ambiental sino de la diversidad de las estructuras económicas y las especificidades de sus ecosistemas.

\section{La reorganización de las instituciones públicas para dar más eficacia a las políticas ambien- tales}

La evaluación de las experiencias de organización institucional pública en materia de medio ambiente ha puesto de manifiesto que el ya tradicional dilema entre una institución sectorial (un ministerio) y una institución transversal (una comisión o consejo) corresponde a un debate superado. Por lo demás, en la práctica este debate ha sido mal planteado, porque varias comisiones se han convertido en organismos sectoriales, diferenciándose de los ministerios sólo por no haber tenido el peso político de éstos.

La discusión debe centrarse entonces en cómo readecuar la institucionalidad para que ésta responda a un conjunto de políticas, debidamente priorizadas, y las políticas ambientales implícitas en las políticas económicas se modifiquen a través de su cruce con las políticas ambientales explícitas. En otras palabras, se hace necesario encontrar la manera de modificar la institucionalidad para minimizar el impacto ambiental negativo de determinadas políticas y disminuir así el carácter reactivo de las políticas ambientales explícitas, coordinándolas con las implícitas.

Para que la solución institucional contribuya con eficiencia a incorporar la dimensión ambiental se requiere la voluntad política del poder legislativo y, en especial, del ejecutivo. Y ante la necesidad de ser pragmáticos en relación con las políticas ambientales, la respuesta institucional debe ser también pragmática.

Tres aspectos básicos aparecen cuando se trata de modificar las estructuras referidas a las políticas ambientales del sector público. El primero dice relación con un quehacer más complejo derivado de la necesidad de hacerse cargo de alguna de las políticas ambientales implícitas, en especial las vinculadas al crecimiento económico; este es el desafío fundamental para los próximos años. La complejidad creciente del tema 
ambiental y la amenaza de que éste se convierta en el talón de Aquiles de las economías de la región obligan a superar la etapa de instituciones públicas que respondan a los problemas del momento, es decir, que sean meramente reactivas. Hay que enriquecer el debate sobre las funciones de las instituciones ambientales de coordinación, por un lado, y las de ejecución, por otro, y abordar seriamente el cruce de las políticas ambientales implícitas en las políticas de desarrollo, especialmente económico, con las políticas ambientales explícitas.

El segundo aspecto se refiere a procesos en los cuales influyen factores exógenos que podrían "obligar" a tomar medidas, aunque no hubiese la voluntad política de hacerlo. Estos factores exógenos son los asociados al comercio internacional y a algunos compromisos internacionales asumidos por cada país. Cada vez más la dimensión ambiental de la gran minería de la región está siendo regulada por las demandas del comercio internacional, y algo similar sucede respecto de algunas importaciones industriales y empieza a aparecer con fuerza en el sector forestal, con la explotación de los bosques nativos.

El tercer aspecto es el de la conciencia de la ciudadanía y la presión poblacional. La creciente contaminación hídrica, atmosférica y de los suelos ha creado conciencia de sus efectos nocivos para el bienestar, lo que se traduce en una presión cada vez mayor de la población por lograr que se resuelvan tales problemas. Por esta razón, los procesos de desarrollo urbano y los de desarrollo industrial están sujetos a exigencias ambientales crecientes. No sucede lo mismo con el espacio rural y el marítimo, donde los procesos son difusos en extensión y algunos de ellos difícilmente perceptibles por ser muy prolongados. Estos procesos deberían recibir una atención preferencial, tanto a través de políticas como de una institucionalidad ambiental especial.

No obstante las consideraciones anteriores, hasta ahora la necesidad de reforzar los organismos ambientales ha llevado en casi todos los países de la región a crear un organismo ambiental central y multifuncional. Normalmente este organismo debe generar políticas y estrategias, y también enfrentar los problemas técnicos cotidianos: es decir, debe cumplir una labor política y una de ejecución de trabajos estrictamente técnicos. La experiencia ha mostrado que en estos casos no se hace bien ni lo uno ni lo otro. Las funciones políticas impulsan a las autoridades de estos organismos a plantear soluciones negociadas que no necesariamente tienen el aval técnico; así, en algunos organismos los informes técnicos son sólo antecedentes que se ponen a disposición de la autoridad ambiental política, la que decide con parámetros obviamente políticos. En consecuencia, pensar en dividir claramente las responsabilidades podría favorecer la incorporación de los aspectos ambientales en los niveles más altos.

Tanto las políticas de ordenamiento ambiental territorial como las relacionadas con las exportaciones y otras de rangos similares son muy difíciles de generar desde un organismo ambiental central como el que se concibe en la mayoría de los países. Convendría entonces que emanaran de una instancia generadora de estrategias ubicada al más alto nivel posible, orientada a incorporar la dimensión ambiental y hacerla parte intrínseca de la modalidad de desarrollo que el país desea impulsar. Es también recomendable que esta instancia no caiga en largas discusiones sobre el desarrollo sustentable, ya que este concepto tan amplio y flexible al que han adherido todos los estamentos de la sociedad suele convertirse en una trampa inmovilista.

La generación de instancias que crucen las políticas implícitas con las explícitas se vería favorecida con una institucionalidad que incluya i) una instancia al más alto nivel para abordar las políticas de desarrollo integral y las políticas ambientales; ii) una instancia técnica independiente de los poderes políticos y de grupos de intereses privados, y iii) una instancia ambiental que incluya a parte de los sectores productivos ligados a los recursos naturales renovables.

Un caso ilustrativo es el de la institucionalidad ambiental pública de México, citada anteriormente. Allí la Secretaría del Medio Ambiente, Recursos Naturales y Pesca tiene unidades relacionadas con la actividad productiva, como la Subsecretaría de Pesca, la Comisión Nacional del Agua y los distintos programas de desarrollo forestal. Y tiene además la Subsecretaría de Planeación, encargada de la articulación intersectorial e intrasectorial, el Instituto Nacional de Ecología y la Procuraduría del Medio Ambiente.

La experiencia mexicana es sin duda un aporte al debate sobre la eficacia de las políticas. El hecho de radicarse en la Subsecretaría de Recursos Naturales la autorización para el manejo de los bosques y las líneas de fomento productivo, permite apoyar y fomentar programas y proyectos realmente sustentables. Pese a los avances logrados, se necesita tiempo para inculcar un sentido ambientalistas a técnicos que en muchos casos han actuado por largo tiempo con sentido productivista de corto plazo. La tarea pendiente de la Secretaría mexicana mencionada es la de infundir las ideas ambientales en los niveles más altos de los sectores tra- 
dicionales de la economía, y ya en la ejecución del programa de medio ambiente de México para los años 1995-2000 se ha progresado al respecto. Lo expuesto lleva a concluir que soluciones como la mexicana, que no puede encasillarse en ninguno de los moldes tradicionales, constituyen interesantes avances hacia la viabilidad de políticas ambientales de reales proyecciones.

\section{Bibliografía}

BID/PNUD (Banco Interamericano de Desarrollo/Programa de las Naciones Unidas para el Desarrollo) (1990): Nuestra propia agenda, Washington, D.C.

Brzovic, F.J. (1989): Crisis económica y medio ambiente en América Latina, LC/R.818, Santiago de Chile, Comisión Económica para América Latina y el Caribe (CEPAL).

CEPAL (1991): El desarrollo sustentable: transformación productiva, equidad y medio ambiente, LC/G.1648 (CONF. 80/2)/Rev.1, Santiago de Chile.

Gligo, N. (1995): Situación y perspectivas ambientales en América Latina y el Caribe, Revista de la CEPAL, $\mathrm{N}^{\circ} 55$, LC/G.1858-P, Santiago de Chile, CEPAL

Gligo, N. y J. Morello (1982): Notas sobre la historia ecológica de América Latina, Estudios Internacionales, año XIII, $\mathrm{N}^{\circ} 49$,
Santiago de Chile, Universidad de Chile, Instituto de Estudios Internacionales.

México, Poder Ejecutivo Federal (1996): Programa de medio ambiente 1995-2000, México, D.F., Talleres Gráficos de México.

PNUMA (Programa de las Naciones Unidas para el Medio Ambiente) (1996): Comparative Study of the Institutional Designs for Environmental Management in the Countries of Latin America and the Caribbean, Nairobi, 7 de octubre.

(1997): Global Environment Outlook, Nairobi.

Venezuela, Ministerio del Ambiente y de los Recursos Naturales Renovables (1982): Sistemas ambientales venezolanos. Informe final. Proyecto VEN/79/001, Caracas. 